Témoigner Témoigner. Entre histoire et mémoire

Getuigen Revue pluridisciplinaire de la Fondation Auschwitz

125 | 2017

Histoire et mémoire de la persécution des

homosexuel-le's par les nazis

\title{
Mick Jackson. Denial (Le procès du siècle)
}

Denial (Mick Jackson)

Jack P. Mener

\section{OpenEdition}

Journals

Édition électronique

URL : https://journals.openedition.org/temoigner/6170

DOI : 10.4000/temoigner.6170

ISSN : 2506-6390

Traduction(s) :

Denial (Mick Jackson) - URL : https://journals.openedition.org/temoigner/6225 [nl]

Éditeur :

Éditions du Centre d'études et de documentation Mémoire d'Auschwitz, Éditions Kimé

Édition imprimée

Date de publication : 1 octobre 2017

Pagination : 22-27

ISBN : 978-2-930953-01-4

ISSN : 2031-4183

Référence électronique

Jack P. Mener, « Mick Jackson. Denial (Le procès du siècle) », Témoigner. Entre histoire et mémoire [En

ligne], 125 | 2017, mis en ligne le 24 décembre 2021, consulté le 03 février 2022. URL : http://

journals.openedition.org/temoigner/6170; DOI : https://doi.org/10.4000/temoigner.6170 
Un moment tentée de suivre les conseils du rabbinat anglais d'éviter le scandale en s'accommodan frontet se fit dófent contes condition 政 A

Anoter que ce retentissant procès qui fit de David rving une vedette, ne fut pas le seul. Il a été plusieur urs condanné, notannent en 2006 à 3 ans de réclucon ferme par le tribunal de Viente pour deux discours prononcés en 1989 (done 17 ans après!) dans as a a àz février 2006). 1 février 2006)

ons déjà quaà l'issue du procès que reconstitue le film Denial, titre traduit en français «Le procès $d u$ ens peut un proc̀s du siccle en la matière, ce fut bien le procès de Nuremberg de 1945-46 intenté par les puissans an

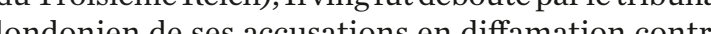
lipdonien de ses accusations en diffamation contre

et dès lors non diffamatoires.

L'intérêt du film Denial et de son scénario, confié au maître du genre David Hare (Licking Hitler, The our...), est davoir suiviavec fidélite et dans sachronoodt fut entraînée piaro dan máné elle. Sa réal Lipstadt fut entrainée presque malgré elle. Sa réalisation des filns de prtoire chers auciném des fins de prétoire chers au cinéna anglo-saxon tout ches choix dun récit linéaire avec un minimum de scènes plus légeres (Lipstadt faisant son jogging, les avocat savonrant d'unon vin leur victoire) favorise plutôt la ment juridique relativement complexe.

Denial offre ainsi une minutieuse et vivante description de ce que fut le procès en diffamation que David Irving déclencha contre le professeur Rache Lipstadt et constitue par là même un précieux out pédagogique a lattention du grand public et des gènéverons détudiás pour comprendre le mécanisme pering pour le combattre.
JOFFRE 1000 DOLLARS

Rappelons que le film débute habilement avec leur emier affrontement direct. Au moment même où Deborah Lipstadt enseige à ses étudints duniversité commentcontrer ave méthode les aru sitéconi nent contreravec méthodes arguments de un hom l'apo minh ce David Irving que vous attaquez daus vo : «e suis ce David Irving que vous attaquez dans vos ouvrage pos Juifs et Isre les Juifs et Israed tirent sigrand proft. Jochre ici, dit-il en agitant une liasse de billets verts, 1000 dollars a quiconque me prouvera que les Juifs ont été gazés à

Le professeur Lipstadt le coupe aussitôt : « Je ne débattrai pas avec vousici, ni maintenant, ni jamais! et sadressant al lassistance: « Je peux discuter sur de opinions, pas sur des faits. L'Holocauste n'est pas une qui nie les fat fin qui nie les faits.» Fin de la discussion donc et de sa la sortie de Cllier une partie de l'assisting àsesayer la sortie de rallier une partie de lassist. Fin aus du $1^{\text {er }}$ as ses ouvrages.

clouer le bec à un interlocuteur de mauvaise foi, un fanatique ou un pervers. Les psys vous le diront: «Comment discuter avec un pervers? Réponse : on ne discute pas. Point.» tla formule vaut pour les négationnistes.

Sauf qu'ici, en procédurier aguerri, David Irving trouva la parade pour reprendre le combat : attirer Lipstadt sur son terrain à lui et l'assigner en diffamation devant une cour britannique. Cest a partir de là que commence un bras de fer juridique serré, complexe Mick Jack re te son scénariste David Hassateu Mick Jack a capter et maintenir l'entière attention du spectateur dans les péripéties juridiques pas toujours faciles à de l'Atlantique.

\section{LE RENVERSEMENT DE LA PREUVE}

ET AUTRES SURPRISES

Revenons à sa première surprise:Lipstadt apprend de son avocat anglais (Anthony Julius, joué avec flegme et sobriété par le relativement jeune mais chevronné acteur irlandais Andrew Scott - Il faut sauver le soldat Ryan, 007 Spectre...) venu à Atlanta la conseille
sur la démarche à suivre que, contrairement au droi américain, en Angleterre c'est elle d'apporter la preuve qu'elle ne ment pas en affirmant que David quand il prétend dans ses propos quand il prétend dans ses propos àgez nont pas exića a gez nont pas te à Auschwitz Centra to tour rister Richard Rampton (joce rister Richard Ranpton (joué Wilkinson) lui dévoile les com Wilkinson) lui devolle les conditions à respecter poun

Qu'elle ne vienne surtout pas

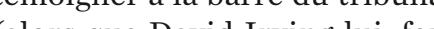
(alors que David Irving lui, feret des médiss, a décidé d’ons lui-même sa propre défense ì lui-même sa propre défense). Lipstadt estomaquée qui estime être compétente pour lui faire . vous. [...] Nous nevousprotégeons pas vous, mais not affaire. Notre stratégie est de nouscentrer sur Irving liseul. Cen'est pas untest devotrecter siltenais de la sien. Tout ce que vous aviez à dire est dans votre l' l..] Ausch de lHolocauste, donc il est au centre même du négationnisme. [...] Nous, nous savons de quoi il s'agit, ma

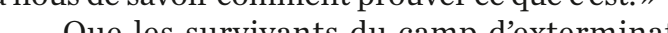

- Que les survivants du camp d'extermination dAuschwitz eux navid losving à la barre. Car David pring, habile débatteur rompu à lexercice du contrénterivé pour les séstabis on ôtant toute crédibilité et validité juidista à leur , otant toute ci

- Qui plus est, la défense acceptera qu'il n'y ait qưun seul juge plutôt que trois, cela pour trancher clairement, et éviter une décision partagée qui pourrait être s'indigner, ce qui importe pour les avocats a beau sour la cause, c'est d'être juridiques avocats et don pour la cause, cest deetre juridqueméc efficaces afin de débouter David Irving par une décision du tribunal qui condanne sans anbiguite le négationnisme à

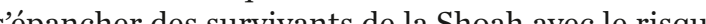

d'un résultat aléatoire, ses défenseurs optent pour la constitution d'un dossier froidement $\mathrm{m}$
sant des faits et des preuves en béton.

Pas de sentimentalisme, des faits, semble être le mot d'ordre de ces juristes hautement expérimentés. «Et si nous perdons, dit-elle, désormais il ser C'estle risque, en effet. C'est dis lors pourqu

Cest des lors pourquoi l'importante équipe de so bureau d'avocats, en consideration de l'enormité de côté de Daner setró et nui n'en saisit pas imces do cote de Dar temén à arpenter nutieusement les lieux.

Ils feront en plus saisir, pour les éplucher page par page,les nombreux mètres linéaires de la bibliothèque de David ningoull a consigné pendant vingt ans dans son journal intime les moindres détails de sa vie. Trade pré rebondissements au cours des a vira, à de précieux

Et c'est précisément sur des détails d'apparence triviale, photos et conclusions scientifiques à l'appui, que le juge de la Haute Cour sera amené à statuer. 
Effectivement, toute l'argumentation de David Irving - qui par ailleurs se défend avec morgue de nêtre ni antisémite ni raciste - va porter sur labsence epres a a chambres a gaz mor

On le sait, avant de fuir le camp d'Auschwitz-Birkenau, les troupes nazies reçurentl'ordre et mirent à exéculion les preuves de leurs crimes, entre guz lont bath

Largum Larionint mass de prétendre que ces ch acolytes négationnistes fut de prètendre que ces chambres à gazn'exts tibles de répandre le typhus. Quant ax phos sceptibles de répandre le typhus. Quant aux photos prise eles n'appes elles n'apportaient apparemment pas la preuve claire t manifeste de l'existence de conduits où, par les

toits, aurait été injecté le fameux gaz mortel Zyklon B (B pour Bayer...).Ce qui incita la presse à sensation présente au procès de titrer en grasà à Une des quotidiens, HOLOCAUST (Pas de trous, pas d'Holocauste).

C'est là où les patients pas d'arpenteur qui avaient permis au barrister Rampton de mesurer sur place les distances entre chambres à gaz et baraquements démontrèrent l'absurdité qu'il y aurait eu à faire accomplir chaque jour par les gardiens nazis d'imporlasts enilles dép fection au Zazklós B contre la vernine. En ous lection au gaz Zyklon B contre la vernine. En outre phiques révélant des traces d’ombres suts photographiques révelant des traces d'ombres sur les toits en pour les conduites de gaz.

Autre péripétie du procès qui contribua à consolider les thèses de la défense : la citation devant la

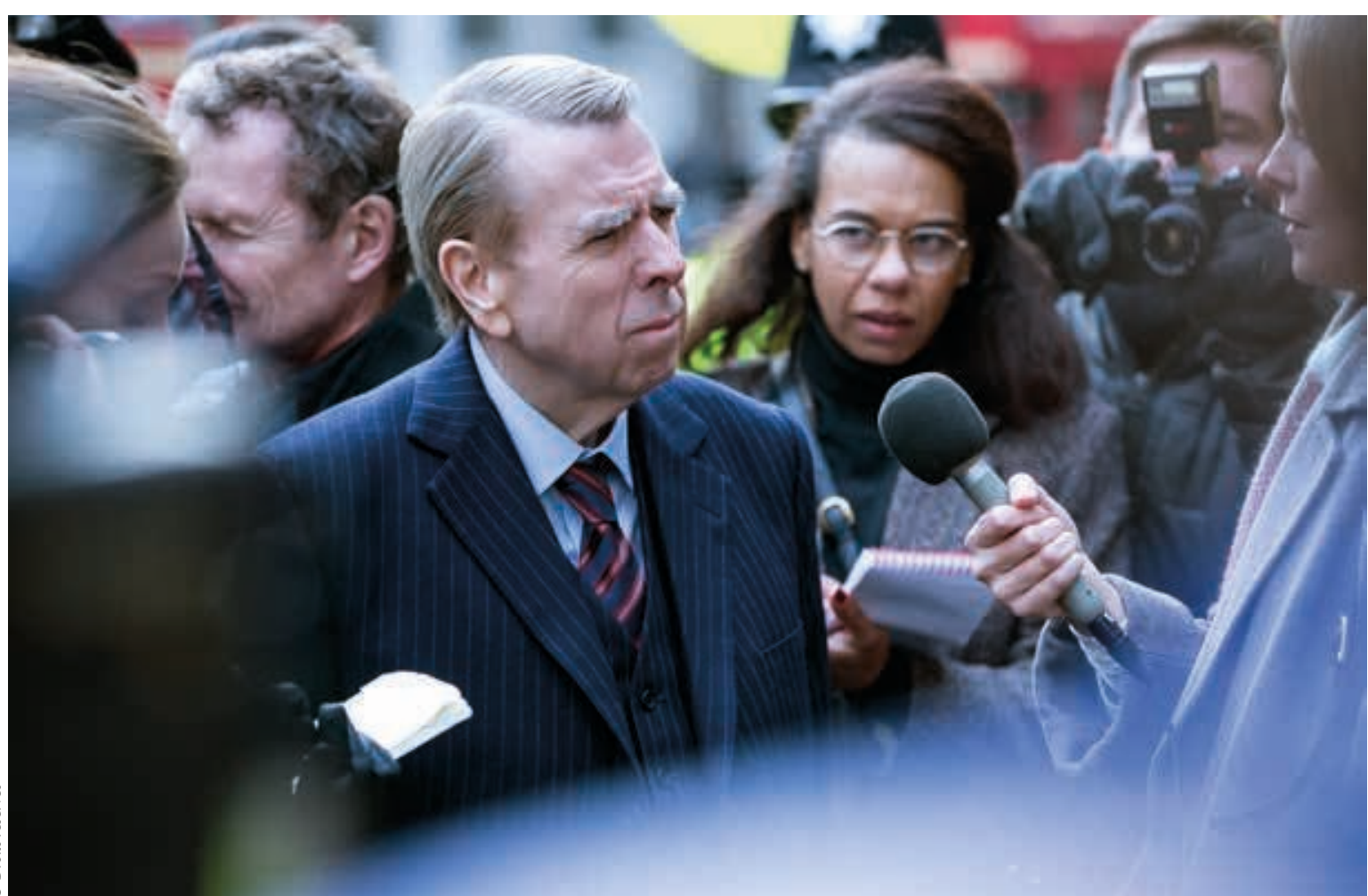

Cour d'extraits du journal intime de David Irving, si acharné à réfuter toute accusation d'antisémitisme et de racisme. Il y relatait qu'il chantait à Jessica, sa fitbete comme: «... Je suis un déper ary Leuser un singe...

Le barnister Rampton eut beau jeu de reprocher Le sentêta Iring - Lisez vos propres Cest cet échange qui perit à láfensed

Cost cetéchange qui permità la défense d'arguer qu'Irvingavait déliberénent Un autre croc-en-jus a négationniste et qui contribuà̀ sa chute. sa chute.

Pour paraphraser l'amusante formule utilisée le 31 janvier 2017 par l'éditorialiste Amanda Marcotte su le site salon.com en réaction à la déclaration de Donal Trump le Jour de la commémoration de la Shoah, Deborah Lipstadt fut contrainte elle aussi de «danser avec le diablen, ce ne fut pas sans délibérément croc-en-ja qui mit David Inving proprement croc-en-jambe qui mit David Irving proprement p
La victoire pour blanchir le professeur Deborah ipstadt de toute accusation ne fut pourtant pas si évidente à remporter.

Le juge Charles Gray au cours des débats, en dépi de son impassibilité, avait donné par moments l'impression quile était sensibleà certains arguments avancés par David Irving. Était-ce par ruse tactique pour mieux débusquer la vérite ? Quoi qu il en soit, aprè

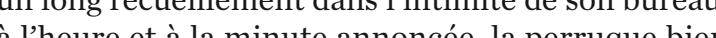
droite, et và la minute annonce, la perruque bien l'innoil il wint solver tho differe pos diffamatoire envers Irving dans ses ouvrages. David Irving avait clairement perdu son défi. In n'est toutefois pas certain que cette conclusion mit fin a juteux commerce de ses nauseabondes publications tion dispars it le de né

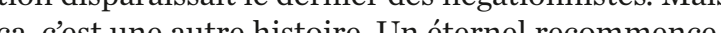
a, cest une autre histoire. Unéternel

Vice-Président de l'Union de la Presse Cinématographique Belge $\rightarrow$ Plus d'infos $\Leftrightarrow$ Denial de Mick Jackson (2016) Avec Rachel Weisz (Deborah Lipstadt), Tom Wilkinson (Richard Rampton), Timothy Spall (David Irving) e.a. 10 minutes. 